\title{
Near-death experiences as a tool for forming a broader comprehension of the link between consciousness and social perception: commentary on Graziano and Kastner (2011)
}

\author{
Christian Agrillo ${ }^{*}$ and Davide Agrillo ${ }^{2}$ \\ Department of General Psychology, University of Padova, Padova, Italy \\ 2 Faculty of Medicine and Surgery, University of Padova, Padova, Italy \\ ${ }^{*}$ Correspondence: christian.agrillo@unipd.it
}

\section{A commentary on}

Human consciousness and its relationship to social neuroscience: a novel hypothesis by Graziano, M. S. A., and Kastner, S. (2011). Cogn. Neurosci. 2, 98-113.

The nature of human consciousness and its neuroanatomical correlates is one of the most profound issues in the field of neuroscience today. Recently, Graziano and Kastner (2011) put forward the intriguing idea that consciousness is a perception, processed by the same neural network which is involved in social perception. The authors provided a number of interesting lines of evidence to support their view, including experiments on the neural correlates of social perception, on neglect, on mirror neurons, and on the mechanisms of decision-making. They also pointed to the involvement of the temporoparietal junction (TPJ) in "out-of-body" experiences. In their words: "given that the right TPJ has been implicated so strongly in the social perception of others, this evidence appears to support the hypothesis that the machinery for social perception also builds a perceptual model of one's own mental experience" (p. 106).

Out-of-body experiences are only one of the recurring features commonly reported within the wider phenomenon known as "near-death experiences" (NDEs). NDEs have been defined as an altered state of consciousness which occurs during an episode of unconsciousness, as a result of a life-threatening condition (Greyson and Stevenson, 1980). Under these circumstances, patients sometimes report perceiving a tunnel, a bright light, deceased relatives, a review of their lives, and out-ofbody experiences, in which they describe a feeling of separation from their bodies and the ability to watch themselves from above. Most patients have described NDEs as very pleasant. Extensive changes in personality are often found to occur after an NDE, such as decrease in the fear of death (Greyson, 1983) and decrease of neurotic anxieties (Noyes and Kletti, 1977). However, some reports of unpleasant experiences similar to nightmares have been also described. Although people have wondered whether "good" people have pleasurable experiences and "bad" people have distressing ones, a lack of correlation between apparent life deeds and type of NDE has been reported (Greyson and Bush, 1992). Interestingly the content of NDEs seems similar worldwide, across cultures and all times: NDEs occur about equally to people of both genders and of all ages, educational and socioeconomic levels, spiritual beliefs, religious affiliations, and life experiences (Bush, 2002). The exact incidence of this phenomenon is not known. However, during the last decade, NDEs are likely to occur with increasing frequency because of the improved techniques of resuscitation (van Lommel, 2011).

According to some authors, NDEs are a transcendental state of consciousness, in which the mind and the self-function work independently from the unconscious body (Greyson, 2000; van Lommel et al., 2001). Both biological and psychological interpretations have been suggested, according to which NDEs would be explained by specific altered mental states related to critical healthy conditions (for recent reviews see Facco, 2010; Agrillo, 2011; Mobbs and Watt, 2011; van Lommel, 2011). For instance, it is known that endorphins are released under stress and are known to block pain and to induce feelings of well-being and even intense pleasure. This might be responsible for the positive emotional tone of most NDEs. In addition, cortical disinhibition associated with anoxia has been considered responsible for the perception of a tunnel (Blackmore, 1996). Regardless of the exact reasons underlying these visions, it is undeniable that NDEs may play an important role in the comprehension of human consciousness.

A large body of evidence has indicated that the temporal lobe is involved in mystical and religious experiences, and a recent study has also found altered temporal lobe functioning in patients who have reported NDEs (Britton and Bootzin, 2004). Temporolimbic epilepsy seems to activate elements of NDEs (Durwen and Linke, 1987), with the involvement of the same neuroanatomical regions which Graziano and Kastner (2011) have suggested form an important neural network for consciousness and social perception.

In addition to neuroanatomical correlates, there is another important aspect that should be mentioned. Many studies have shown that patients sometimes report meeting deceased relatives, friends, or unknown human beings during the time in which they seem to be dying. This has been commonly attributed to the person's expectations or wishes to be reunited with their deceased loved ones at the time of their death. However, if this expectation alone was driving the process, people would presumably recognize the human figures; conversely, most of them are unrecognizable. Several authors have tried to provide an explanation for this incongruence (Agrillo, 2011). It is worth noting that both kinds of vision (familiar and unfamiliar figures) belong to the same category: social stimuli, conspecifics. The deep alteration of consciousness reported in NDEs therefore involves the perception/ hallucination of social stimuli, suggesting a potential link between consciousness and social perception.

Interestingly, many people are emotionally close to their pets and hope to be reunited with them after death. Therefore, in 
accordance with the expectation hypothesis, it would also be reasonable to find a large number of hallucinations of deceased pets (non-social stimuli from an evolutionary perspective). However, Kelly (2001) reported that, among almost 300 cases of NDEs, only two people $(\sim 0.7 \%)$ reported seeing their pets. Again, this is theoretically compatible with the idea of common cognitive-neural systems between our consciousness and the perception of social stimuli.

We believe that Graziano and Kastner (2011) have raised an interesting point, and that the machinery that computes information about other people may be the same machinery that computes information about our own awareness: this would explain why the altered state of consciousness which occurs during NDEs may lead to hallucinations of social stimuli, regardless of people's expectations or wishes.

\section{REFERENCES}

Agrillo, C. (2011). Near-death experience: out-ofbody and out-of-brain? Rev. Gen. Psychol. 15, 1-10.
Blackmore, S. J. (1996). Near-death experiences. J. R. Soc. Med. 89, 73-76.

Britton, W. B., and Bootzin, R. R. (2004). Near-death experiences and the temporal lobe. Psychol. Sci. 15, 254-258.

Bush, N. E. (2002). Afterward: making meaning after a frightening near-death experience. J. Near Death Stud. 21, 99-133.

Durwen,H.F., and Linke, D. B. (1987).Neuropsychological evaluation of patients with temporolimbic epilepsy. Adv. Neurosurg. 15, 152-157.

Facco, E. (2010). Esperienze di premorte. Scienza e coscienza ai confini tra fisica e metafisica. Lungavilla: Edizioni Altravista.

Graziano, M. S. A., and Kastner, S. (2011). Human consciousness and its relationship to social neuroscience: a novel hypothesis. Cogn. Neurosci. 2, 98-113.

Greyson, B. (1983). Near-death experiences and personal values. Am. J. Psychol. 140, 618-620.

Greyson, B. (2000). Dissociation in people who have neardeath experiences: out of their bodies or out of their minds? Lancet 355, 460-463.

Greyson, B., and Bush, N. E. (1992). Distressing neardeath experiences. Psychiatry 55, 95-110.

Greyson, B., and Stevenson, I. (1980). The phenomenology of near death experiences. Am. J. Psychol. 137, 1193-1196.

Kelly, E. W. (2001). Near-death experiences with reports of meeting deceased people. Death Stud. $25,229-249$.
Mobbs, D., and Watt, C. (2011). There is nothing paranormal about near-death experiences: how neuroscience can explain seeing bright lights, meeting the dead, or being convinced you are one of them. Trends Cogn. Sci. (Regul. Ed.) 15, 447-449.

Noyes, R., and Kletti, R. (1977). Panoramic memory: a response to the threat of death. Omega (Westport) 8, 181-194.

van Lommel, P. (2011). Near-death experiences: the experience of the self as real and not as an illusion. Ann. N. Y. Acad. Sci. 1234, 19-28.

van Lommel, P., van Wees, R., Meyers, V., and Elfferich, I. (2001). Near-death experience in survivors of cardiac arrest. Lancet 358, 2039-2045.

Received: 23 November 2011; accepted: 06 January 2012; published online: 23 January 2012.

Citation: Agrillo Cand Agrillo D (2012) Near-death experiences as a tool for forming a broader comprehension of the link between consciousness and social perception: commentary on Graziano and Kastner (2011). Front. Psychology 3:6. doi: 10.3389/fpsyg.2012.00006

This article was submitted to Frontiers in Consciousness Research, a specialty of Frontiers in Psychology.

Copyright (c) 2012 Agrillo and Agrillo. This is an openaccess article distributed under the terms of the Creative Commons Attribution Non Commercial License, which permits non-commercial use, distribution, and reproduction in other forums, provided the original authors and source are credited. 